\title{
The effect of adverse rearing environments on persistent memories in young rats: removing the brakes on infant fear memories
}

\author{
BL Callaghan and R Richardson
}

Mental health problems are often assumed to have their roots in early-life experiences. However, memories acquired in infancy are rapidly forgotten in nearly all species (including humans). As yet, a testable mechanism on how early-life experiences have a lasting impact on mental health is lacking. In these experiments, we tested the idea that infant adversity leads to an early transition into adult-like fear retention, allowing infant memories to have a longer-lasting influence. Rats were exposed to maternal separation ( $3 \mathrm{~h}$ per day) across postnatal days $(\mathrm{P}) 2-14$, or their mother was given corticosterone in her drinking water across the same period. Infant rats were then trained to fear a conditioned stimulus (CS) paired with an aversive unconditioned stimulus (US) on P17. Retention of the fear association was then tested 1-55 days later. When tested one day after the CS-US association was formed, both standard-reared (SR) and maternally-separated (MS) rats exhibited strong memory. However, when tested 10 days later, SR rats exhibited robust forgetting, whereas MS rats exhibited near-perfect retention. These effects were mimicked by exposing the mother to the stress hormone corticosterone in the drinking water. Finally, fear associations in P17 MS rats were retained for up to 30 days. Our findings point to differences in retention of fear as one factor that might underlie the propensity of stress-exposed individuals to exhibit early anxiety symptoms and suggest that manipulations of the corticosterone system may hold the key to ameliorating some of the effects of early stress on persistent retention of fear.

Translational Psychiatry (2012) 2, e138; doi:10.1038/tp.2012.65; published online 10 July 2012

\section{Introduction}

It has long been assumed that mental health problems have their roots in early-life experiences. In particular, modern theories have emphasized the importance of early learning experiences in the development of adult anxiety disorders. ${ }^{1-3}$ However, the widespread belief that early experiences are critical in the development of mental health problems is somewhat paradoxical given that memories formed early in life are rapidly forgotten. ${ }^{4,5}$ Hence, although there are several theories purporting the importance of the early years, few present a testable mechanism on how early-life experiences might have a lasting impact on mental health.

The sparse recollection of early memories is commonly referred to as infantile or childhood amnesia and is a ubiquitous phenomenon occurring in both human and nonhuman animals. ${ }^{4,5}$ For example, humans rarely recall events that occurred before the age of 3 years and generally have 'spotty' recall of experiences between 3 and 6 years of life. ${ }^{5-10}$ The quality of early memories in non-human animals is strikingly similar. For example, rats trained at postnatal day (P) 18 through to P100 were equally apt at forming an association between the black side of a black-white shuttle box and footshock, and passively avoided the black side when tested $24 \mathrm{~h}$ after training. However, after a training-test interval of 1 week, P18 rats were shown to forget rapidly, exhibiting reduced latencies to enter the black side of the shuttle box. Adult rats, in contrast, exhibited virtually perfect retention even when tested as long as 42 days after training. ${ }^{4}$ Although human research has suggested a number of factors that might be responsible for the transition between childhood amnesia and adult-like retention (for example, language acquisition, development of self-concept), preclinical studies have assisted in understanding some of the neurobiological factors associated with infantile forgetting. Specifically, preclinical research has suggested that rather than representing an encoding or storage failure, infantile forgetting typically involves a failure to retrieve the memory. ${ }^{11-13}$ Importantly, it has been suggested that pronounced GABA activity, characteristic of the developing brain, ${ }^{14}$ is responsible for the suppression of these early memories because infantile amnesia can be alleviated by reducing GABA inhibition at test. ${ }^{15}$ Interestingly, it appears that GABA is also involved in memory suppression in adults because enhancing the efficacy of GABA, via benzodiazepine administration, has strong amnestic effects in adult humans and animals, ${ }^{16-18}$ suggesting that infantile forgetting is likely an exaggerated form of adult memory loss.

Although preclinical models of infantile amnesia have been investigated for decades, rarely, if ever, have they been examined in the context of early-life experience. In other

School of Psychology, The University of New South Wales, Sydney, New South Wales, Australia

Correspondence: BL Callaghan, School of Psychology, The University of New South Wales, Level 10, Mathews Building, Sydney, NSW 2052, Australia.

E-mail: b.callaghan@unsw.edu.au

Keywords: development; fear conditioning; infantile amnesia; maternal separation; memory; stress

Received 23 March 2012; revised 1 June 2012; accepted 14 June 2012 
words, we do not know if divergent early-life experiences result in differential retention of infant memories. Considering the proposed importance of infant fear memories for psychiatric functioning, ${ }^{1-3,19}$ and the high degree of comorbidity between psychiatric disorders and specific early-life experiences, for example, adversity, ${ }^{20}$ how such early events influence infant memories seems an important question. Indeed, one unexplored possibility is that exposure to earlylife experiences, such as adversity, may lead to an early transition into the adult-like memory system. If this occurred, memories of infant experiences might be retained for longer, allowing such experiences to have a lasting impact. Importantly, exposure to adversity has already been shown to lead to an early developmental transition in another form of emotional learning-extinction. ${ }^{21}$

At approximately the same time that rats transition into the adult-like memory system, another developmental transition takes place in extinction learning. Specifically, P17 rats typically exhibit a relapse-resistant form of extinction, whereby after extinction training the fear memory cannot be retrieved again. ${ }^{22-24}$ At around $\mathrm{P} 24$, rats make the transition into the adult-like, relapse-prone, extinction system, where a variety of post-extinction manipulations lead to the return of fearful responding (for example, a change of physical context leads to the renewal of fear). In a recent study, it was shown that early experience regulated the developmental transition in extinction. ${ }^{21}$ In that study, rats were removed from their mother for $3 \mathrm{~h}$ per day across days 2-14 of life and then conditioned, extinguished and tested starting at P17. Those rats that were maternally separated (MS) early in development made an early transition from the infant 'relapse-resistant' extinction system to the adult 'relapse-prone' extinction system. Because MS rats exhibit an early developmental shift in extinction, they may also exhibit an early developmental shift in retention of fear memories. In the current series of experiments, we examined this possibility and show that rats subjected to maternal separation early in development exhibit longer retention of fear memories formed during infancy relative to nonstressed controls. Further, we show that this effect is likely mediated by early-life exposure to the stress hormone corticosterone (CORT).

\section{Materials and methods}

Subjects. Experimentally naive Sprague-Dawley-derived rats, bred and housed at the School of Psychology, The University of New South Wales, were used. All rats were 17 days of age at the start of an experiment. The day of birth was designated as P0. Only males were used, and no more than one rat per litter was used per group. Rats were housed with their mother and littermates in plastic boxes $(24.5 \mathrm{~cm}$ long $\times 37 \mathrm{~cm}$ wide $\times 27 \mathrm{~cm}$ high) covered by a wire lid, and food and water were available ad libitum. Animals were treated according to the principals of animal care and use outlined in the Australian Code of Practice for the Care and Use of Animals for Scientific Purposes, ${ }^{25}$ and all procedures were approved by the Animal Care and Ethics Committee at The University of New South Wales.
Maternal separation. During maternal separation (P2-14), all pups were removed from the home cage, weighed and placed in an incubator for $3 \mathrm{~h}$ (starting between 0900 and 1200 hours). The ambient temperature of the incubator was maintained between 27 and $30^{\circ} \mathrm{C}$. Three centimeters of bedding was placed in the incubator so that animals could burrow down for additional warmth if required. Standardreared (SR) animals were exposed to the same handling cues as MS rats (that is, they were briefly weighed each day), but were not removed from the dam for any extended period of time. Using this procedure, we do not see any differences in weight between MS and SR animals at the end of the separation period (that is, at $\mathrm{P} 14$ ). ${ }^{21}$

Drug treatment. In Experiment 3 only, rather than exposing pups to maternal separation, the drinking water of the dams was supplemented with CORT $\left(200 \mu \mathrm{l} \mathrm{ml}^{-1}\right)$ or vehicle $(2.5 \%$ ethanol) across the same period of time (that is, P2-P14). CORT- and vehicle-treated dams consumed an average of 69.05 and $67.35 \mathrm{ml}$ of fluid per day, respectively, across the treatment period and this was not significantly different $\left(t_{19}=0.36, P>0.72\right)$. The dose of CORT was chosen as previous studies demonstrated an increase in basal CORT levels in both lactating mothers and nursing pups following $200 \mu \mathrm{ml}^{-1}$ CORT supplementation in the dam's drinking water. $^{26}$

Apparatus. Rats were trained and tested in a set of two identical chambers that were rectangular $(13.5 \mathrm{~cm}$ long $\times 9 \mathrm{~cm}$ wide $\times 9 \mathrm{~cm}$ high), with the front wall, rear wall and ceiling constructed of clear Plexiglas. The floor and side walls consisted of stainless-steel rods set $1 \mathrm{~cm}$ apart. Two high-frequency speakers were located $8 \mathrm{~cm}$ from either side of the chamber. A custom-built constant-current shock generator could deliver shock to the floor of each chamber as required. Each chamber was housed within a separate wood cabinet so that external noise and visual stimulation were minimized. A red light-emitting diode located on the rear wall was the sole source of illumination in these chambers. A low, constant background noise $(50 \mathrm{~dB}$, measured by a TENMA sound level meter, type 72-860) was produced by ventilation fans located within the cabinet.

Conditioning and test. During conditioning, rats were placed in the context, and after a 2-min adaptation period, a white noise conditioned stimulus (CS) ( $8 \mathrm{~dB}$ above background) was presented for $10 \mathrm{~s}$. The shock unconditioned stimulus (US) $(0.6 \mathrm{~mA}, 1 \mathrm{~s})$ was administered during the last second of the CS (6 pairings given). At test, animals were placed back in the context and, after a 1-min adaptation period, the CS was presented continuously for $2 \mathrm{~min}$.

Scoring, exclusions and statistics. Freezing was scored by a time sampling procedure whereby each rat was scored every $3 \mathrm{~s}$ as freezing or not freezing. Freezing was defined as the absence of all movement except that required for respiration. ${ }^{27}$ These observations were then converted into a percentage score to indicate the proportion of total observations scored as freezing. A second scorer, unaware of the experimental condition of each rat, scored a random 
Table 1 Mean ( \pm s.e.m.) levels of pre-CS freezing at test for MS and SR groups across all experiments

\begin{tabular}{llr}
\hline Experiment & Group & \% Pre-CS freezing \\
\hline 1 & MS-1 day & $25.83(7.06)$ \\
& SR-1 day & $14.00(4.46)$ \\
& MS-10 days & $2.72(2.86)$ \\
& SR-10 days & $1.11(1.17)$ \\
2 & 7 days & $2.5(1.75)$ \\
& 14 days & $0.62(0.67)$ \\
& 30 days & $6.67(5.86)$ \\
& 55 days & $0.00(0.00)$ \\
& Unpaired & $1.25(0.68)$ \\
3 & CORT-1 day & $30.71(9.82)$ \\
& VEH-1 day & $22.00(5.99)$ \\
& CORT-10 days & $5.91(3.86)$ \\
& VEH-10 days & $2.27(1.48)$ \\
& &
\end{tabular}

Abbreviations: CORT, corticosterone; CS, conditioned stimulus; MS, maternally-separated; SR, standard-reared; VEH, vehicle exposed.

sample $(30 \%)$ of all rats tested. The inter-rater reliability was very high across all experiments $(r ' s=0.90-0.96)$.

In Experiments 1 and 3, significant differences in pre-CS freezing at test were detected (see Table 1 for pre-CS freezing levels in all experiments). Because of these differences, CSelicited freezing during test in those experiments were analyzed with analysis of covariance using the pre-CS freezing scores as a covariate. The same results were obtained, however, whether the data were analyzed with analysis of variance or analysis of covariance. When $t$-tests were used, if Levine's test for equality of variances was significant, then the adjusted $t$-statistic and degrees of freedom are reported. Any rats that were statistical outliers at test $(>2.5 \mathrm{~s} . \mathrm{d}$. from the mean) were excluded from subsequent analyses; this resulted in one exclusion from Experiment 3 (vehicle exposed (VEH) -10 days group).

\section{Results}

\section{Experiment 1}

Maternal separation results in longer retention of fear memories in infant rats. Experiment 1 examined the effect of maternal separation on retention of fear memories in P17 rats. It is well established that, under non-stressful conditions, young animals rapidly forget fear associations, ${ }^{15}$ yet it is proposed that early experiences are critical in the development of anxiety disorders. In this experiment, we examined whether infantile amnesia for aversive conditioning could be reduced by exposing rats to an adverse rearing environment-maternal separation.

CS-elicited freezing during test is presented in Figure 1. At the 1-day interval, MS and SR rats exhibited the same high levels of freezing. However, at the 10-day interval, MS rats exhibited much higher levels of freezing than did SR rats. The statistics confirmed this description of the data. There was a significant effect of rearing condition, $F_{1,42}=12.22, P<0.001$, and a significant rearing condition by interval interaction, $F_{1,42}=9.03, P<0.004$. The effect of interval was not significant, $\mathrm{F}_{1,42}=1.03, P=0.32$. Follow-up $t$-tests indicated that MS and SR rats did not differ from one another when tested one day after conditioning, $t_{25}=1.32, P=0.20$, but

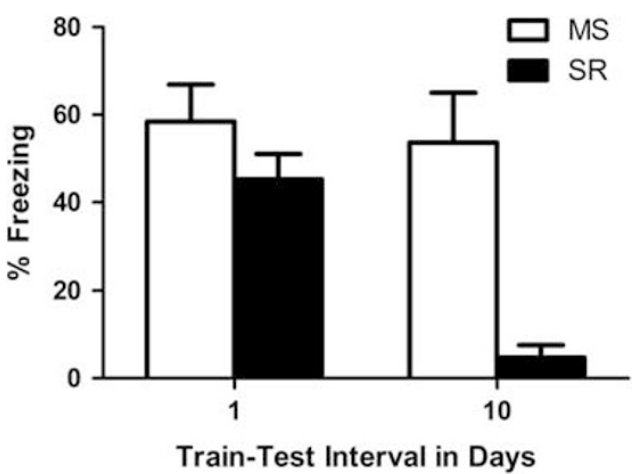

Figure 1 Mean ( \pm s.e.m.) levels of conditioned stimulus (CS)-elicited freezing in maternally-separated (MS; white bars) and standard-reared (SR; black bars) rats tested either 1 or 10 days post-fear conditioning. High and similar levels of fear are present in the MS $(n=12)$ and SR $(n=15)$ rats at the 1-day interval. However, at 10 days post-training, MS $(n=11)$ rats exhibited much higher levels of freezing than $\mathrm{SR}(n=9)$ rats.

were significantly different from one another when tested 10 days after conditioning, $t_{11.26}=4.17, P<0.001$.

\section{Experiment 2}

$M S$ rats retain fear associations for at least 30 days. The results of Experiment 1 support the view that maternal separation results in the development of persistent fear memories in P17 rats. This is a novel finding because infantile amnesia across short retention intervals (a few days) is a very robust phenomenon in non-stressed animals. ${ }^{15,28}$ In Experiment 2, we aimed to replicate the finding that infant MS rats remember for retention intervals of at least a week, and to extend that finding by determining how long they remember the association. We used the same training procedures as in Experiment 1 and then tested the animals at one of four intervals: either 7, 14, 30 or 55 days after training. Because SR rats forgot within the 10-day interval in Experiment 1, and because there are multiple examples of infant rats forgetting a similar fear association within a few days of training, ${ }^{15,28}$ in Experiment 2 we did not include SR animals as a control. Rather, MS rats that were trained to fear the CS were compared to an MS unpaired control group. The unpaired control received the same number of CSs and USs on P17 as the trained rats, but in an explicitly unpaired manner. The unpaired group was then tested for freezing to the CS at one of the various intervals. Using an explicitly unpaired control group allowed us to ask the question: does merely being exposed to footshocks and white noises on P17 lead to high levels of fear responding in MS rats? In other words, we could determine whether the freezing observed in MS rats in Experiment 1 was specific to learning about the CS-US relationship.

There were no differences in levels of freezing to the CS in the unpaired groups tested at the different intervals, $\mathrm{F}_{3,11}=1.79, P=0.23$, hence they were collapsed into a single 'unpaired' group for subsequent analysis. As can be seen in Figure 2, MS paired rats exhibited higher levels of freezing to the CS than 'unpaired' rats at the 7-, 14- and 30day retention intervals, but not at the 55-day retention interval. The statistics confirmed this description of the data. A oneway analysis of variance indicated that there was a significant 


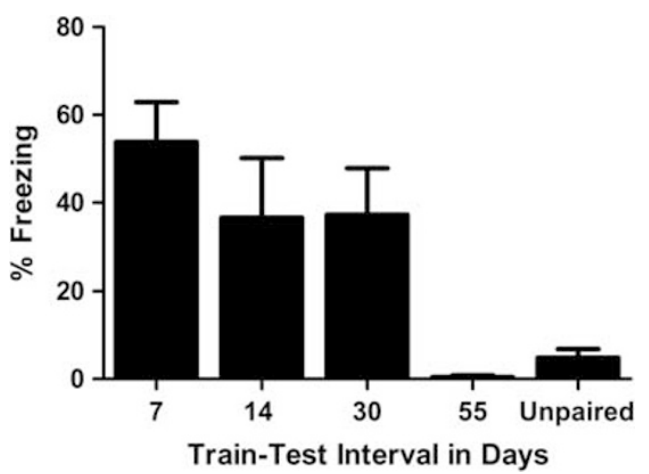

Figure 2 Mean ( \pm s.e.m.) levels of conditioned stimulus (CS)-elicited freezing in maternally-separated rats tested either $7,14,30$ or 55 days post-training, and unpaired rats. Maternally-separated rats that received the paired training exhibited higher levels of fear than unpaired rats at $7(n=8), 14(n=8)$ and $30(n=9)$ days post-training. The 55-day paired group $(n=6)$ exhibited low and similar levels of fear as the unpaired group $(n=12)$.

effect of group, $\mathrm{F}_{4,42}=7.05, P<0.001$. Follow-up tests were performed with the Student-Neuman-Keuls procedure and indicated that the 7-, 14- and 30-day groups were not different from one another $(P>0.35)$ and all were different from the 55-day group and the unpaired group $\left(P^{\prime} s<0.05\right)$, which did not differ from one another.

\section{Experiment 3}

Early-life exposure to CORT results in longer retention of fear memories in infant rats. In Experiment 1, we demonstrated that rats exposed to maternal separation exhibited longer retention of fear memories formed during infancy. In Experiment 2, it was shown that MS rats can retain the fear association formed during infancy for at least 30 days, that is, well into the late adolescent period. These results could be due to a number of factors other than early-life stress (for example, being exposed to a novel context (incubator) during maternal separation, caloric deprivation experienced during the maternal separation period). In Experiment 3, we wanted to examine whether enhanced retention of fear memories in infant MS rats was still seen if maternal separation was replaced with exposing the dams to CORT in the drinking water. That is, can the effects of maternal separation be mimicked by exposing the dam to the stress hormone CORT?

CS-elicited freezing during test is shown in Figure 3. As can be seen, freezing in the CORT-exposed (CORT) and VEH groups did not differ $24 \mathrm{~h}$ after conditioning. However, 10 days after conditioning CORT-exposed rats were freezing at much higher levels than VEH rats. The statistics confirmed this description of the data. There was a significant drug treatment by interval interaction, $F_{1,33}=6.49, P<0.02$. No other effects were significant (largest $\mathrm{F}=0.68, P=0.42$ ). Follow-up $t$-tests indicated that CORT and VEH rats did not differ from one another when tested one day after conditioning, $t_{15}=-0.54$, $P=0.60$, but were significantly different from one another when tested 10 days after conditioning, $t_{14.41}=2.4, P<0.05$.

\section{Discussion}

We have demonstrated that a stressor applied in the early postnatal period, maternal separation, caused infant rats to

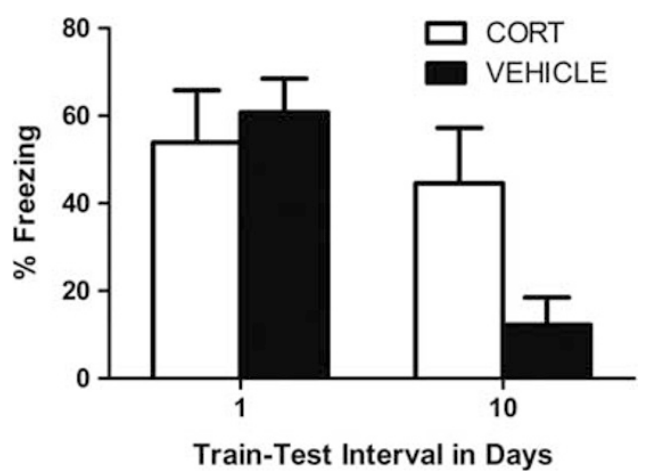

Figure 3 Mean ( \pm s.e.m.) levels of conditioned stimulus (CS)-elicited freezing at test in corticosterone-exposed (CORT; white bars) and vehicle-exposed (VEH; black bars) rats tested either 1 or 10 days post-fear conditioning. At 1 day posttraining, CORT $(n=7)$ and VEH $(n=10)$ rats expressed high and similar levels of fear. However, at 10 days post-training, CORT $(n=11)$ rats exhibited much higher levels of fear than did VEH $(n=10)$ rats.

exhibit better retention of learned fear. Specifically, with our training procedures we replicated previous results demonstrating that non-stressed $\mathrm{P} 17$ rats typically forget a fear association within a 10-day period. ${ }^{15,28}$ Further, we extended those findings to show that stressed $\mathrm{P} 17$ rats exhibit essentially perfect retention of the fear association across that same 10-day period. In addition, we demonstrated that MS rats could retain a fear association formed on $\mathrm{P} 17$ for up to 30 days post-training (that is, MS rats were still expressing fear when they were late adolescents, aged P47). Importantly, the better retention of fear in the MS infant rats was not merely due to these animals always expressing higher levels of freezing because MS rats that did not learn the association between the CS and the US exhibited low levels of fear responding at test (that is, the unpaired control from Experiment 2). Also, these results cannot be explained by differences in rates of learning, because MS and SR rats exhibited similar levels of freezing when tested $24 \mathrm{~h}$ after training. Finally, our results show that early exposure to CORT is sufficient to reproduce these effects, because the offspring of dams given CORT in the drinking water also exhibited better retention. Hence, it would appear that early-life maternal separation or CORT treatment leads infant rats to precociously exhibit adult-like fear retention.

When trained in adulthood, auditory-conditioned fear memories have been shown to be retained across a period of 16 months. For example, adult rats that were given 10 pairings of a tone CS and shock US expressed fear to that CS 480 days later. ${ }^{29}$ In our studies, rats retained fear memories for at least 30 days after conditioning. Although this retention period is not as long as previous demonstrations in adults, it remains novel that infant rats retained fear memories for this period. Importantly, in our studies memories formed in infancy were still expressed in the late adolescent period of development. From infancy to adolescence our rats exhibited approximately a fivefold increase in their weight and physical size. Hence, retaining fear memories across a period of rapid growth is likely a more difficult task than retaining fear memories within a single developmental period such as adulthood. In addition, the conditioning parameters used in the present experiments were weaker than those used in the 
memory retention experiments of adult rats, for example, 6 CS-US pairings in our studies vs 10 CS-US pairings used in the adult retention research, ${ }^{29}$ and to compare directly forgetting functions across ages, initial levels of conditioned fear would need to be consistent.

The fact that infant fear memories were more 'adult-like' following maternal separation is consistent with previous research demonstrating that early-life stress accelerates developmental transitions in other areas. For example, in our previous research we found that the same maternal separation procedure used here resulted in an early transition between the infant and adult extinction systems ${ }^{21}$ and between the adolescent and adult extinction systems. ${ }^{30}$ In those experiments, P17 and P30 rats that had been MS early in life exhibited more adult-like behavior following extinction training than their non-stressed peers. Those results suggested that maternal separation caused a leftward shift in the developmental trajectory of extinction systems, causing later developing systems to come online earlier. Other research groups have also shown accelerated developmental transitions following stress. For example, rats typically show a developmental transition in their behavioral and neural response to an odor previously paired with shock; $\mathrm{P6}-8$ rats do not use the amygdala in this learning task and exhibit a paradoxical approach response to the odor, whereas P10-12 rats do use the amygdala and avoid the odor. ${ }^{31,32}$ Interestingly, after stress or CORT exposure, P6-8 rats precociously exhibit the avoidance response to the shock-paired odor and begin to use the amygdala in that learning task. ${ }^{32,33}$ Importantly, stress has also been shown to accelerate transitions in emotional learning in human children. ${ }^{34}$ In that study, children who had been reared in orphanages (a form of early adversity) were shown to exhibit a more 'adult-like' pattern of amygdala activity to fearful over neutral faces relative to a comparison group of low-adversity exposed children. Taken with these findings, this research suggests that early-life adversity may accelerate the development of the limbic system (for example, amygdala) and the prefrontal cortex, causing the premature emergence of a mature behavioral phenotype.

The fact that maternal exposure to CORT was able to mimic the effects of maternal separation is an important finding for various reasons. Firstly, the procedure of maternal separation is associated with a myriad of factors that act as potential confounding variables; infants are removed from the mother, are exposed to a novel environment, are handled and deprived of calories during the separation period. By exposing dams to CORT in the drinking water, many of these confounding factors are removed. In Experiment 3, being exposed to the stress hormone CORT was sufficient to cause the early transition in fear retention. Although we did not examine maternal behaviors in CORT-treated mothers, there is evidence suggesting that alterations in maternal behavior do occur following CORT treatment. ${ }^{35}$ Hence, the results of Experiment 3 suggest that the CORT-induced alterations in fear retention are the result either of pup CORT exposure through maternal breast milk, altered maternal behavior as a result of CORT exposure or a combination of these factors. Secondly, understanding that early exposure to stress hormones causes a precocious developmental shift in fear retention suggests that the same mechanism might regulate other developmental shifts (for example, in extinction learning). Indeed, as mentioned earlier, CORT exposure has already been shown to be an important regulator of the developmental transition that occurs in infant learning about a shock-paired odor. ${ }^{33}$ Taken with these past results, these data suggest that CORT might act as a general developmental catalyst, forcing the premature maturation of adult functions. Considering that stress-induced maturation of fear and extinction systems appears to increase the risk for anxiety in infant rats, ${ }^{21}$ that is, by enhancing fear retention and relapse following extinction, knowing how stress exerts its effects has considerable clinical utility. For example, reducing infant exposure to stress hormones, both directly and through maternal breast milk, might reduce the likelihood of emerging emotion problems, and can be achieved even in situations when a stressor cannot be avoided.

Consistent with studies that have documented early developmental transitions following stress exposure, some research suggests that stress can accelerate neural development. For example, the same maternal separation procedure used here was recently shown to increase the expression of specific microRNAs (for example, mir132, -124) in the medial prefrontal cortex at $\mathrm{P} 14 .^{36}$ Interestingly, those microRNAs found to be upregulated following maternal separation stress are known to regulate brain maturation in the form of neuronal morphogenesis and differentiation. ${ }^{37,38}$ Hence, it is possible that maternal separation, and other forms of stress early in life, accelerate neuronal development, thereby permitting the expression of behaviors that are reliant on neural circuits that typically develop later. For example, adult rats require a functional prelimbic prefrontal cortex (PL) to express fear memories. ${ }^{39,40}$ Rats begin to express adultlike fear retention at approximately $\mathrm{P} 24^{15}$ and, similar to adult rats, P24 rats cannot express fear when the PL is inactivated. ${ }^{41}$ On the other hand, P17 rats do not require the PL to express fear, and they also do not exhibit adult-like retention of fear memories. ${ }^{15,41}$ In our studies, however, P17 MS rats exhibited excellent retention, characteristic of adult rats. Therefore, one might expect that MS rats, like adult rats, use the PL when expressing fear; we are currently investigating this question.

Another candidate mechanism that might be responsible for the adult-like fear memories seen in infant MS rats is that stress may affect the development of the GABAergic system. Importantly, GABAergic activity is elevated in the developing rat brain ${ }^{14}$ and these high levels of inhibition are considered to be one of the primary factors involved in infantile amnesia. ${ }^{15}$ In other words, it is proposed that adult rats have lower levels of GABAergic inhibition and, therefore, memories are less likely to be suppressed. If maternal separation caused an earlier maturation of the GABAergic system, then endogenous inhibition would be lowered in the P17 rat allowing for the better retention of fear memories, like in adult rats. Interestingly, maternal separation has been shown to increase the expression of brain-derived neurotrophic factor in the PFC of P17 rats $^{42}$ and elevated levels of brain-derived neurotrophic factor have been shown to accelerate the maturation of GABAergic innervation in the cortex. ${ }^{43}$ Hence, it is possible that in this study maternal separation resulted in accelerated 
maturation of the GABAergic system, via a brain-derived neurotrophic factor-dependent mechanism, resulting in less general inhibition and the better retention of memories. Indeed, maturation of GABAergic circuitry in the basolateral amygdala (BLA) has been correlated with the developmental transition seen in extinction learning. ${ }^{44}$ In that study, the maturation of perineuronal nets around GABAergic inhibitory interneurons in the BLA signaled the transition from the infant phenotype of extinction to the adult phenotype of extinction. Of particular interest, those researchers showed that the infant phenotype of extinction could be reactivated in adult rats if perineuronal nets were destroyed in the BLA, via injection of chrondroitinase $A B C$. It would be very interesting to examine whether degradation of perineuronal nets in the BLA would similarly affect fear memory in adult rats. That is, could we reactivate the high levels of forgetting characteristic of infancy via intra-BLA injection of chrondroitinase $A B C$ before fear conditioning in adult rats? This question is an important one, because enhancing the forgetting of fear memories in adults has obvious clinical benefits for groups at high risk of trauma exposure.

Although this research explicitly examined fear memories, it is tempting to speculate about the effects that early-life stress may have on memories of a different valence, for example, appetitive memories. Indeed, if maternal separation were also shown to enhance retention of appetitive memories, this may have implications for substance abuse. Importantly, substance abuse is highly comorbid with anxiety disorders and early-life stress has been shown to increase vulnerability for both addiction and anxiety disorders. ${ }^{20,45,46}$ Considering our present and past findings, that maternal separation increases both the retention of fear and the chance of relapse following fear inhibition, ${ }^{21}$ it is possible that maternal separation may also increase vulnerability to addiction and chance of relapse following extinction of drug taking. Although the impact of maternal separation on appetitive drug taking has already been examined in adults, ${ }^{45}$ to our knowledge, no one has looked at whether maternal separation affects this form of learning in infancy.

In this study, we have shown that maternal separation or CORT exposure results in an early transition between infantile amnesia and the good retention seen in adults. Considering that infantile forgetting is likely an exaggerated form of adult forgetting, ${ }^{15,17}$ further studies examining how infantile forgetting emerges and how it can be manipulated are likely to yield findings relevant across all age groups. Further, our findings point to differences in retention of fear as one factor that might underlie the propensity of stress-exposed individuals to exhibit early anxiety symptoms and suggest that manipulations of the CORT system may hold the key to ameliorating some of the effects of early stress on persistent retention of fear.

\section{Conflict of interest}

The authors declare no conflict of interest.

Acknowledgements. This research was supported by Australian Research Council Discovery Project Grants (DP0985554 and DP120104925) to
RR and an Australian Postgraduate Award to BLC. Financial disclosures: Neither of the authors have any financial disclosures.

1. Jacobs WJ, Nadel L. The first panic attack: a neurobiological theory. Canad J Exp Psychol 1999; 53: 92-107.

2. Mineka S, Oehlberg K. The relevance of recent developments in classical conditioning to understanding the etiology and maintenance of anxiety disorders. Acta Psychol 2008; 127 : 567-580.

3. Mineka S, Zinbarg R. A contemporary learning theory perspective on the etiology of anxiety disorders: it's not what you thought it was. Am Psychologist 2006; 61: 10-26.

4. Campbell BA, Campbell EH. Retention and extinction of learned fear in infant and adult rats. J Comp Physiol Psychol 1962; 55: 1-8.

5. Schulenburg CJ, Riccio DC, Stikes ER. Acquisition and retention of a passive-avoidance response as a function of age in rats. J Comp Physiol Psychol 1971; 74: 75-83.

6. Howes M, Siegel M, Brown F. Early childhood memories: accuracy and affect. Cognition 1993; 47: 95-119.

7. Jack $F$, Hayne $H$. Childhood amnesia: empirical evidence for a two-stage phenomenon Memory 2010; 18: 831-844.

8. Kihlstrom JF, Harackiewicz JM. The earliest recollection: a new survey. J Person 1982; 50: 134-148.

9. Newcombe NS, Lloyd ME, Ratliff KR. Development of episodic and autobiographical memory. A cognitive neuroscience perspective. Adv Child Dev Behav 2007; 35: 40-89.

10. Waldfogel S. The frequency and affective character of childhood memories. Psychol Monogr Gen Appl 1948; 62: i-39.

11. Campbell BA, Jaynes J. Reinstatement. Psychol Rev 1966; 73: 478-480.

12. Richardson R, Riccio DC, Axiotis R. Alleviation of infantile amnesia in rats by internal and external contextual cues. Dev Psychobiol 1986; 19: 453-462.

13. Richardson R, Riccio DC, Jonke T. Alleviation of infantile amnesia in rats by means of a pharmacological contextual state. Dev Psychobiol 1983; 16: 511-518.

14. Frahm C, Draguhn A. GAD and GABA transporter (GAT-1) mRNA expression in the developing rat hippocampus. Dev Brain Res 2001; 132: 1-13.

15. Kim JH, McNally GP, Richardson R. Recovery of fear memories in rats: role of gammaamino butyric acid (GABA) in infantile amnesia. Behav Neurosci 2006; 120: 40-48.

16. Davis M. Diazepam and flurazepam: effects on conditioned fear as measured with the potentiated startle paradigm. Psychopharmacology 1979; 62: 1-7.

17. Harris JA, Westbrook RF. Benzodiazepine-induced amnesia in rats: reinstatement of conditioned performance by noxious stimulation on test. Behav Neurosci 1998; 112: 183-192.

18. Roth T, Roehrs TA. Issues in the use of benzodiazepine therapy. J Clin Psychiatry 1992 53: $14-18$

19. Jacobs W, Nadel L. Stress-induced recovery of fears and phobias. Psychol Rev 1985; 92 512-531.

20. Repetti RL, Taylor SE, Seeman TE. Risky families: family social environments and the mental and physical health of offspring. Psychol Bull 2002; 128: 330-366.

21. Callaghan BL, Richardson R. Maternal separation results in early emergence of adult-like fear and extinction learning in infant rats. Behav Neurosci 2011; 125: 20-28.

22. Kim JH, Richardson R. A developmental dissociation in reinstatement of an extinguished fear response in rats. Neurobiol Learn Mem 2007; 88: 48-57.

23. Kim JH, Richardson R. A developmental dissociation of context and GABA effects on extinguished fear in rats. Behav Neurosci 2007; 121: 131-139.

24. Yap CSL, Richardson R. Extinction in the developing rat: an examination of renewal effects. Dev Psychobiol 2007; 49: 565-575.

25. Australian Government Publishing Service. Australian Code of Practice for the Care and Use of Animals for Scientific Purposes, 7th edn. Australian Government Publishing Service: Canberra, 2004.

26. Catalani A, Marinelli M, Scaccianoce S, Nicolai R, Muscolo LAA, Porcu A et al. Progeny of mothers drinking corticosterone during lactation has lower stress-induced corticosterone secretion and better cognitive performance. Brain Res 1993; 624: 209-215.

27. Fanselow MS. Signaled shock-free periods and preference for signaled shock. $J$ Exp Psychol Anim Behav Process 1980; 6: 65-80.

28. Weber M, McNally GP, Richardson R. Opioid receptors regulate retrieval of infant fear memories: effects of naloxone on infantile amnesia. Behav Neurosci 2006; 120: 702-709.

29. Gale GD, Anagnostaras SG, Godsil BP, Mitchell S, Nozawa T, Sage JR et al. Role of the basolateral amygdala in the storage of fear memories across the adult lifetime of rats. J Neurosci 2004; 24: 3810-3815.

30. Callaghan B, Richardson R. Early-life stress affects extinction during critical periods of development: an analysis of the effects of maternal-separation on extinction in adolescent rats. Stress (in press).

31. Camp LL, Rudy JW. Changes in the categorization of appetitive and aversive events during postnatal development of the rat. Dev Psychobiol 1988; 21: 25-42.

32. Raineki C, Moriceau S, Sullivan RM. Developing a neurobehavioral animal model of infant attachment to an abusive caregiver. Biol Psychiatry 2010; 67: 1137-1145.

33. Moriceau S, Wilson DA, Levine S, Sullivan RM. Dual circuitry for odor-shock conditioning during infancy: corticosterone switches between fear and attraction via amygdala. J Neurosci 2006; 26: 6737-6748. 
34. Tottenham N, Hare T, Millner A, Gilhooly T, Zevin J, Casey B. Elevated amygdala response to faces following early deprivation. Dev Sci 2011; 14: 190-204.

35. Casolini P, Domenici MR, Cinque C, Alemà GS, Chiodi V, Galluzzo M et al. Maternal exposure to low levels of corticosterone during lactation protects the adult offspring against ischemic brain damage. J Neurosci 2007; 27: 7041-7046.

36. Uchida S, Hara K, Kobayashi A, Funato $\mathrm{H}$, Hobara $\mathrm{T}$, Otsuki $\mathrm{K}$ et al. Early life stress enhances behavioral vulnerability to stress through the activation of REST4-mediated gene transcription in the medial prefrontal cortex of rodents. J Neurosci 2010; 30: 15007-15018.

37. Rajasethupathy P, Fiumara F, Sheridan R, Betel D, Puthanveettil SV, Russo JJ et a. Characterization of small RNAs in aplysia reveals a role for miR-124 in constraining synaptic plasticity through CREB. Neuron 2009; 63: 803-817.

38. Vo N, Klein ME, Varlamova O, Keller DM, Yamamoto T, Goodman RH et al. A cAMP. response element binding protein-induced microRNA regulates neuronal morphogenesis. Proc Natl Acad Sci USA 2005; 102: 16426-16431.

39. Corcoran KA, Quirk GJ. Activity in prelimbic cortex is necessary for the expression of learned, but not innate, fears. J Neurosci 2007; 27: 840-844.

40. Sierra-Mercado D, Padilla-Coreano N, Quirk GJ. Dissociable roles of prelimbic and infralimbic cortices, ventral hippocampus, and basolateral amygdala in the expression and extinction of conditioned fear. Neuropsychopharmacology 2010; 36: 529-538.

41. Li S, Kim JH, Richardson R. Differential involvement of the medial prefrontal cortex in the expression of learned fear across development. Behav Neurosci 2012; 126: 217-225.
42. Roceri M, Cirulli F, Pessina C, Peretto P, Racagni G, Riva MA. Postnatal repeated maternal deprivation produces age-dependent changes of brain-derived neurotrophic factor expression in selected rat brain regions. Biol Psychiatry 2004; 55: 708-714.

43. Huang ZJ, Kirkwood A, Pizzorusso T, Porciatti V, Morales B, Bear MF et al. BDNF regulates the maturation of inhibition and the critical period of plasticity in mouse visual cortex. Cell 1999; 98: 739-755.

44. Gogolla N, Caroni $P$, Lüthi $A$, Herry $C$. Perineuronal nets protect fear memories from erasure. Science 2009; 325: 1258-1261.

45. Moffett M, Vicentic A, Kozel M, Plotsky P. Francis D, Kuhar M. Maternal separation alters drug intake patterns in adulthood in rats. Biochem Pharmacol 2007; 73: 321-330.

46. Sinha R. Chronic stress, drug use, and vulnerability to addiction. Ann NY Acad Sci 2008; 1141: $105-130$.

Translational Psychiatry is an open-access journal published by Nature Publishing Group. This work is licensed under the Creative Commons Attribution-Noncommercial-No Derivative Works 3.0 Unported License. To view a copy of this license, visit http://creativecommons.org/licenses/by-nc-nd/3.0/ 